\title{
Natural Variations: New Resources for the Functional Genomics in Rice
}

\author{
Masahiro YANO
}

(National Institute of Agrobiological Sciences)

Artificial mutations showing discrete phenotypes have often been used for analysis of the molecular basis of plant morphology and physiology. In contrast, although a wide range of phenotypic variation is observed among strains or cultivars, these natural variations have not been well analyzed genetically because of their complex inheritance. However, recent progress in plant genome analysis has enabled us to perform comprehensive analysis of such naturally occurring variations. New tools (DNA markers) and information (sequences) have been used for the genetic dissection of complex traits into single genetic factors. Here, we demonstrate that naturally occurring variations are new resources for the functional analysis of rice genes.

Flowering time (heading date) is a major determinant of regional and seasonal adaptation of cultivated rice. Photoperiod and other environmental factors control heading date. A large amount of variation is observed in heading date and photoperiodic response among rice cultivars and strains, including wild relatives. Quantitative trait locus (QTL) analyses of progeny derived from several cross-combinations of cultivars has suggested that more than 15 loci are involved in heading date. In addition, new QTLs have been identified by genetic analysis of a cross between a cultivar and a wild relative. Nearly isogenic lines (NILs) have been developed by marker-assisted selection and used for the analysis of epistatic interaction among QTLs. Map-based cloning has been performed on several QTLs for photoperiodic response. We have demonstrated that $H d I$ is an ortholog of $\mathrm{CO}$ in $\mathrm{Arabi}$ dopsis and is involved in the promotion and inhibition of heading under short-day (SD) and long-day (LD) conditions, respectively. $H d 6$ is involved in inhibition under LD conditions and encodes the alpha-subunit of protein kinase CK2. Hd3a shows a high level of similarity to Arabidopsis FT and functions as a flowering inducer. Ehdl is involved in promotion under SD conditions and encodes a B-type response regulator. Hd5 is involved in inhibition under LD conditions and encodes a putative subunit of a CCAAT-box-binding protein. Lhd 4 is involved in inhibition under LD conditions and encodes a protein with a CCT motif. We have identified several quantitative trait nucleotides (QTNs) that result in loss of function of these QTLs. However, some QTNs defining differences at the level of function remain to be clarified. Combining information from genetic and sequencing analyses has revealed that combinations of natural alleles with loss or gain of function at particular QTLs, such as $H d 1, H d 5, H d 6$, Ehd1, and Lhd4, seem to generate a wide range of continuous variation in photoperiodic flowering in rice. These genetic and molecular analyses have allowed us to propose a genetic pathway controlling photoperiodic flowering in rice. Identification and expression analyses of genes suggest that there are both conserved and divergent features in photoperiodic control of flowering in rice, an SD plant, and Arabidopsis, an LD plant.

Marker-based genetic approaches can also be used to analyze other complex traits besides flowering time. Several primary mapping populations, including backcross inbred lines (BILs) and doubled-haploid lines, have been developed to facilitate the analysis of complex traits. Several QTLs have been identified with major effects on such characteristics as seed dormancy, cool-temperature tolerance at the booting stage, seed shattering habit, seed longevity, and UV-B tolerance. Marker-assisted genetic approach greatly facilitates the exploitation of agronomically and scientifically important genes in natural variants. In order to mine scientifically or agronomically important alleles, we have been developing novel mapping populations, such as chromosome segment substitution lines. In these lines, a particular chromosome segment from a donor line is substituted in the genetic background of the recurrent line. The substituted segments cover all chromosomes in a whole set of lines. Their potential power in genetic analysis has been demonstrated in plants. These materials allow detailed and reliable QTL analyses.

Marker-assisted approaches have contributed to our understanding of a wide range of complex traits in rice, including plant morphology and environmental stress tolerance. These approaches have enabled us not only to identify genetically the genes involved in complex traits, but also to identify them at the molecular level. In addition, the advent of DNA marker technology has made it possible to establish new breeding strategies, such as marker-assisted breeding. That strategy requires comprehensive dissection and understanding of complex traits of interest. Once we have identified tightly linked markers, NILs for target QTLs with 
minimum introgressed chromosomal segments from donor cultivars can be readily and quickly developed by marker-assisted selection. In addition, we will be able to introduce genes derived from different donor cultivars into elite rice cultivars by using a variety of
NILs and DNA markers (trait pyramiding). This strategy has not yet been applied in phenotype-based selection in rice breeding. Genome sequence information has served, and will continue to serve, as a launching pad for these achievements.

\title{
U.S. Plant Science Research: Trends and Future Directions
}

\author{
Machi Dilworth
}

(National Science Foundation, USA)

The twenty-first (21 $\left.{ }^{\text {st }}\right)$ Century has been called "the Century of Biology". Twenty-first Century Biology is multidisciplinary, multi-dimensional, information-rich, education-oriented and internationally engaged. With the advent of genomics and recent advances in information technology, high-resolution imaging and analytical instrumentation, and high-throughput methodologies, biology is evolving rapidly. Plant science is no exception. Recent advances, trends and future directions in plant sciences will be discussed within this context.

Advances in plant sciences in the United States have accelerated with the establishment of the National Plant Genome Initiative (NPGI) in 1998 as a coordinated national plant genome research program. Research was initially focused on building research tools that would enable the entire scientific community to participate in the genomics revolution. As a result, basic tools for plant genomics are now widely available, including: whole genome sequences of Arabidopsis, rice, poplar, and several plant associated microbes; transposon-tagged mutant collections; informatics tools and databases; and techniques such as TILLING and microarray-based expression profiling. While development of genomics research tools continues, the research focus is shifting towards the use of these tools to answer major questions in plant biology in such wide-ranging topics as epigenetics, cell wall biosynthesis, polyploidy, chemical ecology, and biodiversity. In addition, plant scientists are taking advantage of tools and concepts being developed in all fields of sciences. Structural biology, nanotechnology, imaging technologies, highthroughput analytical instrumentation, information sciences, and mathematics, are being added to the plant science toolbox. In the process, plant science has been invigorated and has attracted a new crop of scientists. Current research is leading plant sciences to a higher level where it may be possible to begin to understand every molecular interaction in every cell of a plant throughout its life cycle, and to construct a wiring diagram for the plant body. A big roadblock facing the plant science community is how best to manage the enormous amount and disparate kinds of plant biology data accumulating at an alarming rate all over the world.

In an editorial published in March 1998 (Science, 279, 2019), Philip Abelson declared, "The scientific world is in an early phase of a genomics revolution, a third technological revolution following the Industrial Revolution and the computer-based revolution". He further stated, "The greatest ultimate global impact of genomics will result from manipulation of the DNA of plants .... Today, humans employ the capabilities of only a few plants. A major challenge is to explore the opportunities inherent in some of the hundreds of thousands of them". Six years after that prediction, the plant genomics revolution is in full force. Plant scientists all over the world are working to meet the challenge. Many notable advances in plant sciences were made possible by international collaboration. Continued and increased communication among the world plant scientists will strengthen their efforts and accelerate advances for the benefit of all.

[Any opinions, findings, conciusions, or recommendations expressed in this talk are those of the author, and do not necessarily represent the official views, opinions, or policy of the National Science Foundation.] 